\title{
Use of a levonorgestrel-releasing intrauterine device for menorrhagia treatment during adjuvant therapy of adrenocortical carcinoma with mitotane
}

\author{
Piotr Robert Szkodziak, Piotr Czuczwar, Sławomir Woźniak, \\ Filip Szkodziak, Tomasz Paszkowski \\ $3^{\text {rd }}$ Chair and Department of Gynecology, Medical University of Lublin, Poland
}

Adrenocortical carcinoma is a rare tumour with high malignancy and poor prognosis. This tumour is rarely diagnosed in the reproductive age. Complete surgical resection is the only curative treatment for adrenal cancer in all stages. After surgery adjuvant chemotherapy is required. Mitotane is the most important drug in adrenal cancer chemotherapy. Mitotane's mode of action is not entirely explained. Animal studies have shown that the substance exerts a direct cytotoxic effect on the cells of the adrenal cortex. This activity is selective, progressive and affects only the zona reticularis and fasciculata of the adrenal cortex. Mitotane inhibits cortisol synthesis by disrupting the chain of cholesterol. It has been suggested, that mitotane also affects the peripheral metabolism of steroids, especially of transcortin (CBG). This results in an increase of $C B G$ blood concentration and a reduction of the amount of free hormones.

During adrenal cancer adjuvant therapy mitotane is used for 4-5 years in the non-invasive tumours, while in the invasive stages the treatment is continued for life. High toxicity is the main concern with mitotane treatment, which may result, inter alia, from the coagulopathy.

A 33 year old patient underwent right-sided adrenalectomy because of a non-functional adrenocortical carcinoma (the non-invasive form). After the procedure the patient was qualified for corticosteroid replacement and adjuvant mitotane therapy. After 10 months of treatment menorrhagia occurred. Transvaginal ultrasound (TVUS) was performed (Fig. 1). Due to irregular appearance of the endometrium, the patient was qualified for a hysteroscopy with endometrial biopsy. The histopathological result revealed simple endometrial hyperplasia. After this procedure, menorrhagia did not subside and the patient was hospitalized again with a diagnosis of sideropenic anemia, which required a blood transfusion and intravenous and oral iron supplementation. The lowest haemoglobin ( $\mathrm{Hb}$ )

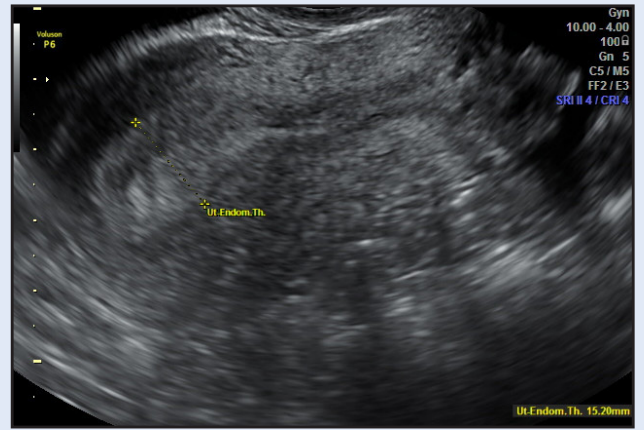

Figure 1. TVUS performed on $10^{\text {th }}$ cycle day, before hysteroscopy. Endometrium was thickened and heterogeneous.

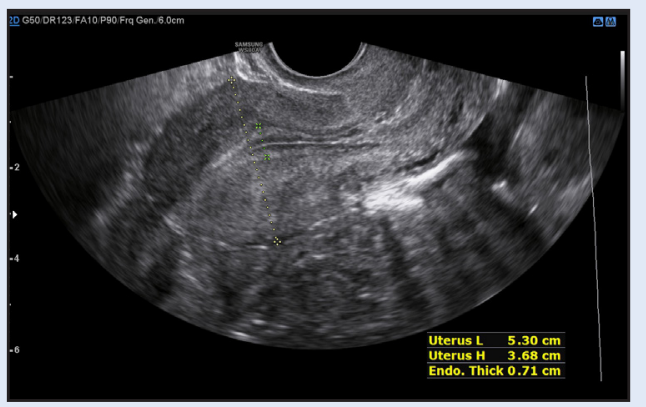

Figure 2. TVUS performed on $10^{\text {th }}$ cycle day, 12 months after LNG-IUD removal. Normal thickness and echogenicity of endometrium. level was $7.5 \mathrm{~g} \%$. To prevent simple endometrial hyperplasia and associated clinical consequences, the patient was qualified for levonorgestrel-releasing intrauterine device (LNG-IUD). The patient was followed-up for 4 years until the end of adjuvant therapy with mitotane. At this time menorrhagia and/or 
metrorrhagia were not observed. Hb values were normal, LNG-IUD tolerance was good. No influence of LNG-IUD on the mitotane effectiveness and tolerance has been observed.

LNG-IUD was removed 5 years after the insertion and 1 year after ending mitotane therapy. Both TVUS (Fig. 2) and the histopathological result of an endometrial biopsy performed 12 months after removal of the LNG-IUD were normal.

The pathogenesis of menorrhagia during mitotane therapy may be mainly explained by influence on peripheral hormones metabolism, while coagulopathy only exacerbates clinical bleeding symptoms. Increased CBG binds free progesterone and ultimately leads to a relative hyperestrogenism. This explains the occurrence of simple endometrial hyperplasia 10 months after introducing adjuvant mitotane therapy, and a satisfying response to LNG-IUD treatment. 